\title{
On Extensions of the Alon-Tarsi Latin Square Conjecture
}

\author{
Daniel Kotlar* \\ Department of Computer Science \\ Tel-Hai College \\ Upper Galilee 12210, Israel \\ dannykot@telhai.ac.il
}

Submitted: Apr 19, 2012; Accepted: Oct 10, 2012; Published: Oct 25, 2012

Mathematics Subject Classifications: 15A15, 05B15

\begin{abstract}
Expressions involving the product of the permanent with the $(n-1)^{\text {st }}$ power of the determinant of a matrix of indeterminates, and of (0,1)-matrices, are shown to be related to an extension to odd dimensions of the Alon-Tarsi Latin Square Conjecture, first stated by Zappa. These yield an alternative proof of a theorem of Drisko, stating that the extended conjecture holds for Latin squares of odd prime order. An identity involving an alternating sum of permanents of $(0,1)$-matrices is obtained.
\end{abstract}

Keywords: Latin square, Alon-Tarsi Latin Square conjecture, Parity of a Latin square, adjacency matrix, permanent of $(0,1)$-matrix.

\section{Introduction}

A Latin square of order $n$ is an $n \times n$ array of numbers in $[n]:=\{1, \ldots, n\}$ so that each number appears exactly once in each row and each column. Let $L_{n}$ be the number of Latin squares of order $n$. Let $\operatorname{Sym}(n)$ be the symmetric group of permutations of $[n]$. For a permutation $\pi \in \operatorname{Sym}(n)$ we denote its sign by $\epsilon(\pi)$. Viewing the rows and columns of a Latin square $L$ as elements of $\operatorname{Sym}(n)$, the row-sign (column-sign) of $L$ is defined to be the product of the signs of the rows (columns) of $L$. The sign of $L$, denoted $\epsilon(L)$, is the product of the row-sign and the column-sign of $L$. The parity of a Latin square is even (resp. odd) if its sign is 1 (resp. -1). The row parity and column parity of a Latin square are defined analogously. We denote by $L_{n}^{\mathrm{EVEN}}\left(L_{n}^{\mathrm{ODD}}\right)$ the number of even (odd) Latin

*The author thanks an anonymous reviewer for providing the proofs of Theorem 7 and Corollary 1. 
squares of order $n$. The Alon-Tarsi Latin Square Conjecture [1] asserts that for even $n$, $L_{n}^{\mathrm{EVEN}}-L_{n}^{\mathrm{ODD}} \neq 0$. Values of $L_{n}^{\mathrm{EVEN}}-L_{n}^{\mathrm{ODD}}$ for small $n$ can be found in [10]. Drisko [3] proved the conjecture for $n=p+1$, where $p$ is an odd prime, and Glynn [5] proved it for $n=p-1$. Since for odd $n$ it holds that $L_{n}^{\text {EVEN }}=L_{n}^{\mathrm{ODD}}$, some extensions of this conjecture that are applicable to odd $n$ were proposed, as will be described shortly.

A Latin square is called normalized if its first row is the identity permutation. A Latin square is called unipotent if all the elements of its main diagonal are equal. Let $U_{n}^{\mathrm{E}}$ (resp. $U_{n}^{\mathrm{O}}$ ) be the numbers of even (resp. odd) Latin squares of order $n$ which are both normalized and unipotent. Zappa [12] defined the Alon-Tarsi constant $A T(n):=U_{n}^{\mathrm{E}}-U_{n}^{\mathrm{O}}$ and proposed the following extension of the Alon-Tarsi conjecture:

Conjecture 1. For all $n, A T(n) \neq 0$.

A Latin square is called reduced if its first row and first column are both equal to the identity permutation. Let $R_{n}^{\mathrm{E}}$ and $R_{n}^{\mathrm{O}}$ denote the numbers of even and odd reduced Latin squares of order $n$, respectively. Another possible extension of the Alon-Tarsi conjecture was recently stated in [10]:

Conjecture 2. For all $n, R_{n}^{\mathrm{E}}-R_{n}^{\mathrm{O}} \neq 0$.

If $n$ is even these two conjectures are equivalent to the Alon-Tarsi conjecture. However, despite the existence of a bijection between reduced Latin squares and normalized unipotent Latin squares of order $n$ (see [12]), it is not clear whether for odd $n$ the two conjectures are equivalent. Drisko [4] proved Conjecture 1 in the case that $n$ is an odd prime. Conjecture 2 is only known to be true for small values of $n$ (see [10]).

A Latin square $L$ of order $n$ determines $n$ permutation matrices $P_{s}, s \in[n]$, defined by $\left(P_{s}\right)_{i j}=1$ if and only if $L_{i j}=s$. Let $S_{n}$ be the collection of all $n \times n$ permutation matrices. For $P \in S_{n}$ let $\alpha_{P}$ be the corresponding permutation in $\operatorname{Sym}(n)$. The symbol-sign of $L$, denoted by $\epsilon_{\mathrm{sym}}(L)$, is the product of all the $\epsilon\left(\alpha_{P_{s}}\right), s=1, \ldots, n$. A Latin square $L$ is symbol-even if $\epsilon_{\mathrm{sym}}(L)=1$ and symbol-odd if $\epsilon_{\mathrm{sym}}(L)=-1$.

Let $X=\left(X_{i j}\right)$ be the $n \times n$ matrix of indeterminates. The following theorem is due to MacMahon [7]:

Theorem 1. $L_{n}$ is the coefficient of $\prod_{i=1}^{n} \prod_{j=1}^{n} X_{i j}$ in $\operatorname{per}(X)^{n}$.

Here $\operatorname{per}(A)$ denotes the permanent of $A$. Stones [9] showed that if we replace permanent by determinant in the expression in Theorem 1, an expression for the Alon-Tarsi conjecture is obtained:

Theorem 2. $L_{n}^{\mathrm{EVEN}}-L_{n}^{\mathrm{ODD}}$ is the coefficient of $(-1)^{n(n-1) / 2} \prod_{i=1}^{n} \prod_{j=1}^{n} X_{i j}$ in $\operatorname{det}(X)^{n}$.

The idea of taking the $n^{\text {th }}$ power of the determinant was used by Stones [9] to obtain another expression for $L_{n}^{\mathrm{EVEN}}-L_{n}^{\mathrm{ODD}}$ :

Theorem 3. Let $B_{n}$ be the set of all $n \times n(0,1)$-matrices. For $A \in B_{n}$ let $\sigma_{0}(A)$ be the number of zero elements in $A$. Then

$$
L_{n}^{\mathrm{EVEN}}-L_{n}^{\mathrm{ODD}}=(-1)^{\frac{n(n-1)}{2}} \sum_{A \in B_{n}}(-1)^{\sigma_{0}(A)} \operatorname{det}(A)^{n} .
$$


It will be shown in Section 2 that when $n$ is odd "hybrid" expressions involving one permanent and $n-1$ determinants yield analogous results for $A T(n)$. Section 3 contains an alternative proof of Drisko's result [4], that $A T(p) \neq 0$ for all odd primes $p$. In Section 4 a formula linking Conjectures 1 and 2 is obtained. Section 5 introduces a formula relating the permanents of all distinct regular $p \times p$ adjacency matrices of bipartite graphs (up to renaming the vertices of one of the sides).

\section{Formulae for $A T(n)$}

For $\alpha \in \operatorname{Sym}(n)$ let $L_{n}^{\mathrm{SE}}(\alpha)$ (resp. $\left.L_{n}^{\mathrm{SO}}(\alpha)\right)$ be the number of symbol-even (resp. symbolodd) Latin squares with $\alpha=\alpha_{P_{1}}$. Let $L_{n}^{\mathrm{CE}}(\alpha)$ (resp. $L_{n}^{\mathrm{CO}}(\alpha)$ ) be the number of columneven (resp. column-odd) Latin squares with $\alpha$ as the first column. Let $L_{n}^{\mathrm{CE}}(\alpha, \beta)$ (resp. $\left.L_{n}^{\mathrm{CO}}(\alpha, \beta)\right)$ be the number of column-even (resp. column-odd) Latin squares with $\alpha$ as the first row and $\beta$ as the first column. We have

Lemma 1. If $n$ is odd then

$$
\sum_{\pi \in \operatorname{Sym}(n)} \epsilon(\pi)\left(L_{n}^{\mathrm{SE}}(\pi)-L_{n}^{\mathrm{SO}}(\pi)\right)=(-1)^{\frac{n(n-1)}{2}} n !(n-1) ! A T(n) .
$$

Proof. Viewing a Latin squares as a set of $n^{2}$ triples $(i, j, k)$, such that $L_{i j}=k$, and applying the mapping $\tau:(i, j, k) \rightarrow(i, k, j)$, the $k^{\text {th }}$ column of $\tau(L)$ is the permutation $\alpha_{P_{k}}$ corresponding to the permutation matrix $P_{k}$ in $L$. Thus $L_{n}^{\mathrm{SE}}(\alpha)=L_{n}^{\mathrm{CE}}(\alpha)$ and $L_{n}^{\mathrm{SO}}(\alpha)=L_{n}^{\mathrm{CO}}(\alpha)$. We have

$$
\sum_{\pi \in \operatorname{Sym}(n)} \epsilon(\pi)\left(L_{n}^{\mathrm{SE}}(\pi)-L_{n}^{\mathrm{SO}}(\pi)\right)=\sum_{\pi \in \operatorname{Sym}(n)} \epsilon(\pi)\left(L_{n}^{\mathrm{CE}}(\pi)-L_{n}^{\mathrm{CO}}(\pi)\right) .
$$

By applying $\pi^{-1}$ to the columns of each Latin squares with $\pi$ as its first column we see that if $n$ is odd then $\epsilon(\pi)\left(L_{n}^{\mathrm{CE}}(\pi)-L_{n}^{\mathrm{CO}}(\pi)\right)=L_{n}^{\mathrm{CE}}(\mathrm{id})-L_{n}^{\mathrm{CO}}(\mathrm{id})$. Thus,

$$
\sum_{\pi \in \operatorname{Sym}(n)} \epsilon(\pi)\left(L_{n}^{\mathrm{SE}}(\pi)-L_{n}^{\mathrm{SO}}(\pi)\right)=n !\left(L_{n}^{\mathrm{CE}}(\mathrm{id})-L_{n}^{\mathrm{CO}}(\mathrm{id})\right) .
$$

Since exchanging columns of a Latin square does not alter the column parity we have that for each $\beta \in \operatorname{Sym}(n)$ such that $\beta(1)=1, L_{n}^{\mathrm{CE}}(\beta, \mathrm{id})-L_{n}^{\mathrm{CO}}(\beta, \mathrm{id})=L_{n}^{\mathrm{CE}}(\mathrm{id}$, id $)-$ $L_{n}^{\mathrm{CO}}(\mathrm{id}, \mathrm{id})$. Thus,

$$
\begin{aligned}
\sum_{\pi \in \operatorname{Sym}(n)} \epsilon(\pi)\left(L_{n}^{\mathrm{SE}}(\pi)-L_{n}^{\mathrm{SO}}(\pi)\right) & =n !\left(L_{n}^{\mathrm{CE}}(\mathrm{id})-L_{n}^{\mathrm{CO}}(\mathrm{id})\right) \\
& =n ! \sum_{\substack{\beta \in \operatorname{Sym}(n) \\
\beta(1)=1}} L_{n}^{\mathrm{CE}}(\beta, \mathrm{id})-L_{n}^{\mathrm{CO}}(\beta, \mathrm{id}) \\
& =n !(n-1) !\left(L_{n}^{\mathrm{CE}}(\mathrm{id}, \mathrm{id})-L_{n}^{\mathrm{CO}}(\mathrm{id}, \mathrm{id})\right)
\end{aligned}
$$


We use the notation $R_{n}^{(+,-)}$for the number of reduced Latin squares with even row parity and odd column parity $\left(R_{n}^{(+,+)}, R_{n}^{(-,+)}\right.$and $R_{n}^{(-,-)}$are defined accordingly). Since $L_{n}^{\mathrm{CE}}(\mathrm{id}, \mathrm{id})$ is the number of column-even reduced Latin squares, we have:

$$
\begin{aligned}
L_{n}^{\mathrm{CE}}(\mathrm{id}, \mathrm{id})-L_{n}^{\mathrm{CO}}(\mathrm{id}, \mathrm{id}) & =R_{n}^{(+,+)}+R_{n}^{(-,+)}-R_{n}^{(+,-)}-R_{n}^{(-,-)} \\
& =R_{n}^{(+,+)}-R_{n}^{(-,-)} .
\end{aligned}
$$

Since

$$
A T(n)=\left\{\begin{array}{lll}
R_{n}^{(+,+)}-R_{n}^{(-,-)}, & \text {if } n \equiv 0,1 \quad(\bmod 4) \\
R_{n}^{(-,-)}-R_{n}^{(+,+)}, & \text {if } n \equiv 2,3 \quad(\bmod 4),
\end{array}\right.
$$

by Section 5 in [12], the result follows.

We now have a result, analogous to Theorem 2, for $A T(n)$ :

Theorem 4. Let $n$ be odd and let $X=\left(X_{i j}\right)$ be the $n \times n$ matrix of indeterminates. Then $A T(n)$ is the coefficient of $(-1)^{\frac{n(n-1)}{2}} \prod_{i=1}^{n} \prod_{j=1}^{n} X_{i j}$ in $\frac{1}{n !(n-1) !} \operatorname{per}(X) \operatorname{det}(X)^{n-1}$.

Proof. For $\mathcal{P} \in\left(S_{n}\right)^{n}$ let $\mathcal{P}=\left(P_{1}, P_{2}, \ldots, P_{n}\right)$ and for $s=1, \ldots, n$ let $\alpha_{s}=\alpha_{P_{s}}$. Expanding $\operatorname{per}(X)$ and $\operatorname{det}(X)$ we obtain

$$
\operatorname{per}(X) \operatorname{det}(X)^{n-1}=\sum_{\pi \in \operatorname{Sym}(n)} \prod X_{i \pi(i)} \sum_{\substack{\mathcal{P} \in\left(S_{n}\right)^{n} \\ \pi=\alpha_{1}}} \prod_{s=2}^{n} \epsilon\left(\alpha_{s}\right) \prod_{k=1}^{n} X_{k \alpha_{s}(k)} .
$$

Now, for each $\pi \in \operatorname{Sym}(n)$ the coefficient of $\prod_{i=1}^{n} \prod_{j=1}^{n} X_{i j}$ in

$$
\prod X_{i \pi(i)} \sum_{\substack{\mathcal{P} \in\left(S_{n}\right)^{n} \\ \pi=\alpha_{1}}} \prod_{j=2}^{n} \epsilon\left(\alpha_{j}\right) \prod_{i=1}^{n} X_{i \alpha_{j}(i)}
$$

is equal to $\epsilon(\pi)\left(L_{n}^{\mathrm{SE}}(\pi)-L_{n}^{\mathrm{SO}}(\pi)\right)$. Hence, by (2.1), the coefficient of $\prod_{i=1}^{n} \prod_{j=1}^{n} X_{i j}$ in $\operatorname{per}(X) \operatorname{det}(X)^{n-1}$ is

$$
\sum_{\pi \in \operatorname{Sym}(n)} \epsilon(\pi)\left(L_{n}^{\mathrm{SE}}(\pi)-L_{n}^{\mathrm{SO}}(\pi)\right)
$$

and the result follows from Lemma 1.

We also have an analogue of Theorem 3 for $A T(n)$ :

Theorem 5. Let $B_{n}$ be the set of all $n \times n(0,1)$-matrices. For $A \in B_{n}$ let $\sigma_{0}(A)$ be the number of zero elements in $A$. If $n$ is odd then

$$
A T(n)=\frac{(-1)^{\frac{n(n-1)}{2}}}{n !(n-1) !} \sum_{A \in B_{n}}(-1)^{\sigma_{0}(A)} \operatorname{per}(A) \operatorname{det}(A)^{n-1}
$$


Proof. Most of the proof follows Stones' proof of Theorem 3. By (2.1),

$$
\sum_{A \in B_{n}}(-1)^{\sigma_{0}(A)} \operatorname{per}(A) \operatorname{det}(A)^{n-1}=\sum_{(A, \mathcal{P}) \in B_{n} \times\left(S_{n}\right)^{n}} Z(A, \mathcal{P}),
$$

where

$$
Z(A, \mathcal{P})=(-1)^{\sigma_{0}(A)} \prod_{i=1}^{n} A_{i \alpha_{1}(i)} \prod_{s=2}^{n} \epsilon\left(\alpha_{s}\right) \prod_{k=1}^{n} A_{k \alpha_{s}(k)} .
$$

If for $(A, \mathcal{P})$ there exists $i, j \in[n]$ such that $\left(P_{s}\right)_{i j}=0$ for all $s=1, \ldots, n$, then let $A^{c}$ be the matrix formed by toggling $A_{i j}$ in the lexicographically first such coordinate $i j$. Thus $Z(A, \mathcal{P})=-Z\left(A^{c}, \mathcal{P}\right)$ and these two terms cancel in the sum in (2.3). So, on the right hand side of (2.3) we are left only with $\sum_{\mathcal{P} \in S^{*}} \prod_{s=2}^{n} \epsilon\left(P_{s}\right)$, where $S^{*}=\left\{\left(P_{1}, \ldots, P_{n}\right)\right.$ : $\sum_{s=1}^{n} s P_{s}$ is a Latin square $\}$ and $A$ is the all-1 matrix. Now,

$$
\begin{aligned}
\sum_{\mathcal{P} \in S^{*}} \prod_{s=2}^{n} \epsilon\left(\alpha_{s}\right) & =\sum_{\pi \in \operatorname{Sym}(n)} \epsilon(\pi) \sum_{\substack{\mathcal{P} \in S^{*} \\
\alpha_{P_{1}}=\pi}} \prod_{s=1}^{n} \epsilon\left(\alpha_{s}\right) \\
& =\sum_{\pi \in \operatorname{Sym}(n)} \epsilon(\pi) \sum_{\substack{\mathcal{P} \in S^{*} \\
\alpha_{P_{1}}=\pi}} \epsilon_{\mathrm{sym}}\left(\sum_{s=1}^{n} s P_{s}\right) \\
& =\sum_{\pi \in \operatorname{Sym}(n)} \epsilon(\pi)\left(L_{n}^{\mathrm{SE}}(\pi)-L_{n}^{\mathrm{SO}}(\pi)\right),
\end{aligned}
$$

and the result follows from Lemma 1.

\section{An alternative proof of Drisko's theorem}

The main result of this section (Corollary 1) was first proved by Drisko [4]. An alternative proof, based on the results of Section 2, is presented here. I am indebted to an anonymous reviewer for suggesting this proof.

In this section the rows and columns of an $n \times n$ matrix will be indexed by the numbers $0,1, \ldots, n-1$.

Definition 1. Let $A$ be an $n \times n$ matrix and Let $B$ be a subset of cells of $A$. Let $k$ be an integer. The $k$-left shift of $B$ is the set of cells $\left\{b_{i,(j-k) \bmod n}: b_{i, j} \in B\right\}$. The $k$-down shift of $B$ is the set of cells $\left\{b_{(i+k) \bmod n, j}: b_{i, j} \in B\right\}$.

Definition 2. An $n \times n$ matrix $A$ will be said to be $k$-left row shifted, for some $k$, $0<k<n$, if for all $i=1, \ldots, n-1$, the $i^{\text {th }}$ row of $A$ is equal to the $k$-left shift of the $(i-1)^{\text {st }}$ row, and the $0^{\text {th }}$ row is equal to the $k$-left shift of the $(n-1)^{\text {st }}$ row.

Remark 6 . If $p$ is an odd prime and $A$ is a $p \times p k$-left row shifted matrix, then the set of cells of $A$ is the disjoint union of $p$ diagonals, where the elements of each diagonal are all equal. These diagonals will be referred to as the k-broken diagonals of $A$. 
Lemma 2. Let $A$ be a $p \times p$ k-left row shifted (0,1)-matrix, where $p$ is an odd prime. Let $\mathbf{b}$ be the first row of $A$ and let $|\mathbf{b}|$ be the number of 1's in $\mathbf{b}$. Then

(i) $\operatorname{per}(A) \equiv|\mathbf{b}|(\bmod p)$

(ii) $\operatorname{det}(A) \equiv \pm|\mathbf{b}|(\bmod p)$

Proof. Part (i) can be easily obtained from Ryser's permanent formula ([8], see also http://mathworld.wolfram.com/RyserFormula.html). However, a different approach, that will also apply to Part (ii), is used here. We define a mapping $s_{k}$ on the set of diagonals of $A$ as follows: For a diagonal $d$ in $A, s_{k}(d)$ is obtained by taking the $k$-left shift of $d$ and then taking the 1-down shift of the result. Note that the fixed points of $s_{k}$ are exactly the $k$-broken diagonals defined in Remark 6 . The mapping $s_{k}$ is a bijection and, since $A$ is $k$-left row shifted, $s_{k}(d)$ contain the same set of values as $d$. In particular, if $d$ consists only of 1 's, so does $s_{k}(d)$. Also note that $s_{k}^{p}(d)=d$ for all $d$ and thus, since $p$ is prime, each orbit under $s_{k}$ is of size 1 or $p$. As mentioned above, the orbits of size 1 are those containing the $k$-broken diagonals. Thus, $\operatorname{per}(A) \bmod p$ is equal to the number of $k$-broken diagonals consisting only of 1's, and since there are $|\mathbf{b}|$ such diagonal Part (i) follows.

For Part (ii), we need to show that $s_{k}$ preserves the parity of the permutation corresponding to the diagonal acted upon, and that all $k$-broken diagonals correspond to permutations of the same parity. Let $d_{1}$ and $d_{2}$ be two diagonals. Suppose that $d_{1}$ is the $l$-left shift of $d_{2}$ for some $l$. This means that if $\pi_{1}$ and $\pi_{2}$ are the corresponding permutations, then $\pi_{2}=\nu^{l} \circ \pi_{1}$ (application from right to left), where $\nu=(12 \ldots p)$. Since $p$ is odd, $\nu$ is an even permutation, and thus $d_{1}$ and $d_{2}$ correspond to permutations of the same parity. If $d_{1}$ is the $l$-down shift of $d_{2}$, then the corresponding permutations satisfy $\pi_{1}=\pi_{2} \circ \nu^{l}$. Since $s_{k}$ consists of a left shift and a down shift, $s_{k}$ preserves the parity. Now suppose $d_{1}$ and $d_{2}$ are $k$-broken diagonals. Then $d_{1}$ is the $l$-left shift of $d_{2}$ for some $l$. As shown above, $d_{1}$ and $d_{2}$ correspond to permutations of the same parity. It follows that all fixed diagonals correspond to permutations of the same parity. This proves (ii).

Theorem 7. Let $B_{p}$ be the set of all $p \times p(0,1)$-matrices, where $p$ is an odd prime. Then

$$
\frac{1}{p} \sum_{A \in B_{p}}(-1)^{\sigma_{0}(A)} \operatorname{per}(A) \operatorname{det}(A)^{p-1} \equiv 1 \quad(\bmod p) .
$$

Proof. Define the group $G=\langle\nu\rangle \times\langle\nu\rangle$, where $\nu=(12 \cdots p)$. The group $G$ acts on $B_{p}$ by permuting the rows and columns, so that for each element of $G$, its first component permutes the order of the rows and the second component permutes the order of the columns. By The Orbit-Stabilizer Theorem, an orbit has size $|G|=p^{2}$ unless each of its elements has a non-trivial stabilizer in $G$. If $g=\left(\nu^{i}, \nu^{j}\right)$ is a stabilizer of $A \in B_{p}$, so is any of its powers, including $\left(\nu, \nu^{k}\right)$ for some $k$, since $p$ is prime. Thus, an orbit has size smaller than $p^{2}$ if and only if for each matrix $A$ in that orbit there exists some $0<k<p$ for which $\left(\nu, \nu^{k}\right) A=A$. Let

$$
D=\left\{A \in B_{p} \mid\left(\nu, \nu^{k}\right) A=A \text { for some } 0<k<p\right\} .
$$


The action of $G$ preserves $\sigma_{0}$ and, since $\nu$ is an even permutation, it also preserves the permanent and the determinant. We have

$$
\frac{1}{p} \sum_{A \in B_{p}}(-1)^{\sigma_{0}(A)} \operatorname{per}(A) \operatorname{det}(A)^{p-1} \equiv \frac{1}{p} \sum_{A \in D}(-1)^{\sigma_{0}(A)} \operatorname{per}(A) \operatorname{det}(A)^{p-1} \quad(\bmod p) .
$$

Hence, it suffices to prove (3.1) with " $B_{p}$ " replaced by " $D$ ".

Suppose $\left(\nu, \nu^{k}\right) A=A$. Then, after applying $\nu^{k}$ to the $i^{\text {th }}$ row the $(i+1)^{\text {st }}$ is obtained, for $i=0, \ldots, p-2$ and applying $\nu^{k}$ to the $(p-1)^{\text {st }}$ row yields the $0^{\text {th }}$ row. This implies that $A$ is a $(p-k)$-left row shifted matrix. Thus, $A$ is uniquely determined by its first row $\mathbf{b}$ and the number $k$. We denote this by $A=A(\mathbf{b}, k)$.

Now, suppose $A=A(\mathbf{b}, k)$ is not the all-1 matrix and let $a=|\mathbf{b}|$. Since $p$ is odd, $\sigma_{0}(A) \equiv a+1(\bmod 2)$. Then, by Lemma 2 and Fermat's Little Theorem, $(-1)^{\sigma_{0}(A)} \operatorname{per}(A) \operatorname{det}(A)^{p-1} \equiv-\left((-1)^{a} a\right)(\bmod p)$. For a fixed $a \in\{1, \ldots, p-1\}$, the number of distinct matrices $A(\mathbf{b}, k)$ with $|\mathbf{b}|=a$ is $\left(\begin{array}{l}p \\ a\end{array}\right)(p-1)$. Therefore,

$$
\frac{1}{p} \sum_{A \in D}(-1)^{\sigma_{0}(A)} \operatorname{per}(A) \operatorname{det}(A)^{p-1} \equiv-\frac{1}{p} \sum_{a=1}^{p-1}\left(\begin{array}{l}
p \\
a
\end{array}\right)(p-1)(-1)^{a} a \quad(\bmod p),
$$

where the cases that $a \in\{0, p\}$ have been discarded since they correspond to the all-0 and all-1 matrices, which have zero determinant. The result now follows from the binomial identity

$$
\sum_{a=0}^{p}\left(\begin{array}{l}
p \\
a
\end{array}\right)(-1)^{a} a=0
$$

(see http://en.wikipedia.org/wiki/Binomial_coefficient).

The following result was first proved by Drisko [4]:

Corollary 1. If $p$ is an odd prime, then

$$
A T(p) \equiv(-1)^{\frac{p-1}{2}} \quad(\bmod p) .
$$

Proof. When $n=p$ is an odd prime we can rearrange (2.2) to obtain

$$
\begin{aligned}
A T(p) & =(-1)^{\frac{p-1}{2}} \times \frac{1}{(p-1) !^{2}} \times \frac{1}{p} \sum_{A \in B_{p}}(-1)^{\sigma_{0}(A)} \operatorname{per}(A) \operatorname{det}(A)^{p-1} \\
& \equiv(-1)^{\frac{p-1}{2}}(\bmod p),
\end{aligned}
$$

by Wilson's theorem and Theorem 7. The result follows. 


\section{Linking Conjectures 1 and 2}

The following statement is obtained as part of a proof in [6]:

Proposition 1. Let $n$ be odd and let $A_{1}, A_{2}, \ldots, A_{n}$ be $n \times n$ matrices over a field. Then

$$
\sum_{\substack{\rho, \sigma \in \operatorname{Sym}(n)^{n} \\ \rho_{1}=\mathrm{id}}} \epsilon\left(\sigma_{1}\right) \epsilon(\sigma) \epsilon(\rho) \prod_{i, j=1}^{n}\left(A_{j}\right)_{\sigma_{i}(j), \rho_{j}(i)}=(n-1) ! \cdot\left(R_{n}^{\mathrm{E}}-R_{n}^{\mathrm{O}}\right) \operatorname{per}\left(A_{1}\right) \prod_{j=2}^{n} \operatorname{det}\left(A_{j}\right) .
$$

Here $\rho_{1}$ and $\sigma_{1}$ are the first components in $\rho$ and $\sigma$ respectively. Combining Proposition 1 with Theorem 4 yields the following identity, linking $A T(n)$ and $R_{n}^{\mathrm{E}}-R_{n}^{\mathrm{O}}$ :

Theorem 8. Let $X=\left(X_{i j}\right)$ be an $n \times n$ matrix of indeterminates. Then $A T(n) \cdot\left(R_{n}^{\mathrm{E}}-R_{n}^{\mathrm{O}}\right)$ is the coefficient of $(-1)^{\frac{n(n-1)}{2}} \prod_{i=1}^{n} \prod_{j=1}^{n} X_{i j}$ in

$$
\frac{1}{n !(n-1) !^{2}} \sum_{\substack{\rho, \sigma \in \operatorname{Sym}(n)^{n} \\ \rho_{1}=\mathrm{id}}} \epsilon\left(\sigma_{1}\right) \epsilon(\sigma) \epsilon(\rho) \prod_{i, j=1}^{n} X_{\sigma_{i}(j), \rho_{j}(i)}
$$

Proof. This follows by taking $A_{1}=A_{2}=\cdots=A_{n}=X$ in (4.1) and applying Theorem 4 .

Thus, showing that the above coefficient is nonzero would prove Conjectures 1 and 2 .

\section{On the permanent of adjacency matrices}

The evaluation of the permanent of a $(0,1)$-matrix is of special significance, since it was the first proven \#P-complete problem. This was shown by Valiant in a landmark paper ([11], see also [2]). Theorem 5 leads to an interesting identity involving the permanents of certain (0,1)-matrices:

Theorem 9. Let $p$ be an odd prime, let $B_{p}$ be the set of $p \times p(0,1)$-matrices, and let $B_{p}^{*}=\left\{A \in B_{p}: \operatorname{det}(A) \not \equiv 0(\bmod p)\right\}$. Let $B_{p}^{\dagger}$ be a set of representatives in $B_{p}$ of the row permutation classes. Then

$$
\sum_{A \in B_{p}^{\dagger} \cap B_{p}^{*}}(-1)^{\sigma_{0}(A)} \operatorname{per}(A) \equiv-1 \quad(\bmod p) .
$$

Proof. Let $B_{p}^{r}$ be the subset of $B_{p}$ containing the regular matrices. From (2.2) we have:

$$
A T(p)=\frac{(-1)^{\frac{p-1}{2}}}{p !(p-1) !} \sum_{A \in B_{p}^{r}}(-1)^{\sigma_{0}(A)} \operatorname{per}(A) \operatorname{det}(A)^{p-1}
$$

If $A^{\prime}$ can be obtained from $A$ by permuting the rows, then $\operatorname{per}\left(A^{\prime}\right)=\operatorname{per}(A)$ and $\operatorname{det}\left(A^{\prime}\right)^{p-1}=\operatorname{det}(A)^{p-1}$ (since $p-1$ is even). Since the rows of each $A \in B_{p}^{r}$ are all 
distinct, each row permutation class in $B_{p}^{r}$ contains exactly $p$ ! matrices. Let $B_{p}^{\dagger}$ be a set of representatives of the row permutation classes in $B_{p}$. Then

$$
A T(p)=\frac{(-1)^{\frac{p-1}{2}}}{(p-1) !} \sum_{A \in B_{p}^{\dagger} \cap B_{p}^{r}}(-1)^{\sigma_{0}(A)} \operatorname{per}(A) \operatorname{det}(A)^{p-1} .
$$

By Fermat's little theorem and Wilson's theorem we have

$$
A T(p) \equiv(-1)(-1)^{\frac{p-1}{2}} \sum_{A \in B_{p}^{\dagger} \cap B_{p}^{*}}(-1)^{\sigma_{0}(A)} \operatorname{per}(A) \quad(\bmod p) .
$$

The result follows from Corollary 1.

Remark 10. If we view an $n \times n(0,1)$-matrix $A$ as the adjacency matrix of a bipartite graph $G_{A}$, having two parts of identical size $n$, then $\operatorname{per}(A)$ is the number of perfect matchings in $G_{A}$. A set $B_{p}^{\dagger}$, as in Theorem 9, represents all possible such graphs, up to renaming the vertices of one of the parts.

\section{References}

[1] N. Alon and M. Tarsi, Colorings and orientations of graphs, Combinatorica 12 (1992), no. 2, 125-134.

[2] A. Ben-Dor and S. Halevi, Zero-one permanent is \#p-complete, a simpler proof, Theory and Computing Systems, 1993., Proceedings of the 2nd Israel Symposium on the, IEEE, 1993, pp. 108-117.

[3] A. A. Drisko, On the number of even and odd Latin squares of order $p+1$, Advances in Math. 128 (1997), 20-35.

[4] A. A. Drisko, Proof of the Alon-Tarsi conjecture for $n=2^{r} p$, The Electronic Journal of Combinatorics (1998), no. R28.

[5] D. Glynn, The conjectures of Alon-Tarsi and Rota in dimension prime minus one, SIAM J. Discrete Math. 24 (2010), 394-399.

[6] D. Kotlar, A weak case of Rota's basis conjecture for odd dimensions, manuscript (arXiv:1110.1830v3).

[7] P. A. MacMahon, A new method in combinatory analysis, with applications to Latin squares and associated questions, Trans. Cambridge Phil. Soc. 16 (1898), 262-290.

[8] H. J. Ryser, Combinatorial mathematics, The Mathematical Association of America, 1963.

[9] D. S. Stones, Formulae for the Alon-Tarsi conjecture, SIAM J. Discrete Math. 26 (2012), no. 1, 65-70.

[10] D. S. Stones and I. M. Wanless, How not to prove the Alon-Tarsi conjecture, Nagoya Math. J. 205 (2012), 1-24. 
[11] L.G. Valiant, The complexity of computing the permanent, Theoretical Computer Science 8 (1979), no. 2, 189-201.

[12] P. Zappa, The Cayley determinant of the determinant tensor and the Alon-Tarsi conjecture, Advances in Applied Mathematics 19 (1997), 31-44. 